\title{
Review of Restorable Routing Algorithm in Optical Networks
}

\author{
Navneet Kaur \\ Department of Computer \\ Science and Engineering \\ DAVIET College \\ Jalandhar $(\mathrm{Pb})$
}

\author{
Raman Kumar \\ Department of Computer \\ Science and Engineering \\ DAVIET College \\ Jalandhar $(\mathrm{Pb})$
}

\begin{abstract}
Restorability of a network refers to a network's capability to provide continuous service in the presence of failures and how a network restores to the actual position after overcoming a failure. In a WDM network, a single channel may be carrying no of gigabits of data per second, a single failure may cause a disturbance in huge amount of service for a large number of users. A network that is built on fiber-optics technology sends data digitally, through connected fiber. Optical networks increase the Transmission capacity and speed of the network. This paper reviews most of the recent research work on Routing Algorithms in optical Networks. Various Routing Algorithms are discussed in the Literature.
\end{abstract}

\section{Keywords}

OLTs, OADMs, OXCs, Bandwidth, Optical fiber, Optical network

\section{INTRODUCTION}

An optical network provides a common infrastructure over which a variety of services can be delivered. These networks are also capable of delivering bandwidth in a flexible manner when needed. Optical fibers are very thin glass cylinders which carries signal in the form of light or (optical signals). Optical fibers offer much higher bandwidth than copper cables and other undesirable effects. As a result, it is preferred medium for transmission of data at anything more than a few tens of megabits per second over any distance more than a kilometer. It is also preferred medium of realizing shortdistance, high-speed interconnections inside large systems. There are many ways to increase the capacity of the optical fiber and one of the ways is wavelength division multiplexing (WDM).

A light path is an "optical communication path" between two nodes, established by allocating the same wavelength throughout the route of the transmitted data [1]. A light path is uniquely identified by a wavelength and a physical path. Two light paths can use the same fiber link, if and only if they use different wavelengths. If two nodes are connected by a light path, a message can be sent from one node to the other without requiring any buffering [2].

\subsection{Optical Network Elements}

Optical Network Architecture includes different following elements:

Optical line terminals (OLTs): Optical Line Terminal Multiplex multiple wavelengths into a single fiber \& demultiplex wavelengths on a single fiber into separate wavelengths.

Optical add/drop multiplexers (OADMs): OADMs Add/Drop one or more wavelengths to make a composite WDM signal.
Optical cross connects (OXCs): OXCs switch the wavelengths from one port to another.

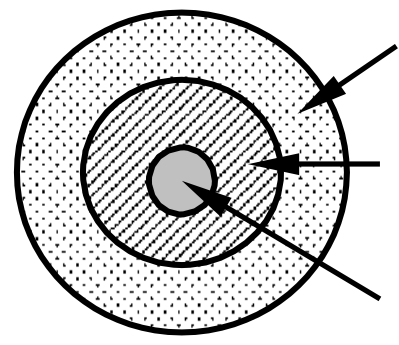

Fig 1: Cross-section of an optical fiber

\subsection{Routing Algorithm}

A routing algorithm establishes the paths that messages must follow to reach their destination. used. Routing algorithms can be broadly classified into three types:

Fixed Routing, Alternate Routing and Exhaust Routing.

In the Fixed Routing, for every node pair only one candidate route is provided. This candidate route for node pair is fixed and does not change with changing network traffic.

In the Alternate Routing, for every node pair a set of $\mathrm{K}$ candidate routes are provided. The set of candidate routes are provided for a node pair is a subset of all possible routes. When a connection request arrives for a pair $\mathrm{p}$, one of the candidate routes is selected

In Exhaust Routing, there is no restriction on the selecting a route, for a given node pair $\mathrm{p}$, a route among all possible routes is chosen

\subsection{Blocking Probability}

The blocking probability [2] of a light path request is an important performance measure of a wavelength-routed network. The blocking probability is affected by many factors such as network traffic load, structure of the network. A network containing wavelength converters has a better call blocking performance than a network without wavelength converters. Wavelength conversion is an extremely costly technology, it may not be economical to install wavelength converters at every node of a network. Rather, it may be more efficient to put wavelength converters at some, but not all nodes in the network called sparse wavelength-conversion network.

\section{REROUTING SCHEME FOR RESTORABLE NETWORKS}

Wavelength rerouting is a technique which is used to reduce the bandwidth loss that is caused by wavelength continuity constraint in wavelength routed network. Wavelength rerouting shifts few existing light paths to new wavelengths without changing their route. It moves a few existing light 
paths to new wavelengths so as to create a wavelengthcontinuous route to satisfy a new connection request. Wavelength rerouting is applicable to networks with dynamic traffic demand. It is also useful in case of failure of any network component. When a network component such as a node or link fails all light paths which currently uses that node or link fail. To restore the service on these light paths s new light paths needs to be established between end nodes of failed light paths s. In such a scenario, wavelength rerouting of unaffected light paths $s$ helps to restore the failed light paths s. Wavelength rerouting basically has two components, light paths migration and the rerouting algorithm. The rerouting operation deals with migration of the light paths. It is desirable that a rerouting operation incurs shorter disruption time and simplifies switching control at routing nodes. The rerouting algorithm determines light paths that can be rerouted and selects few among them to create a wavelengthcontinuous route to satisfy a connection request. It is desirable that a rerouting algorithm should be simple, run in polynomial time and minimize the number of existing light paths requests to be rerouted. The Authors consider wavelength rerouting procedure as a possible approach to reduce network shortcoming arisen from wavelength continuity constraint.

Rerouting algorithms may generally be categorized as follows:

\subsection{Passive rerouting}

In Passive Rerouting only if the simple routing procedure fails then rerouting procedure tries to accommodate the new connection request by shifting some existing light paths $[9,11]$.

\subsection{Active rerouting}

In this rerouting procedure is typically controlled by a timer and it shifts/migrates existing light paths/connections to better routes periodically [21]

\subsection{Light path level rerouting}

The traffic of light paths at the full wavelength capacity granularity is rerouted $[9,11]$.

\subsection{Connection level rerouting}

The traffic of connections at different bandwidth granularities are rerouted [21].

\section{ROUTING ALGORITHMS}

There are a number of wavelength routing techniques proposed in literature. The important routing schemes considered in literature.

\subsection{WLCR-FF (Weighted Least Congested Routing- First fit)}

WLCR-FF is a type of fixed alternate routing algorithm. In the Fixed Alternate Routing, for every node pair p, a set of S candidate routes are provided. In fixed alternate routing routes are searched one by one in a pre-determined order. Usually, these routes are ordered in non-decreasing order. fixed alternate routing scheme have been proposed with the objective of minimizing the blocking probability for dynamically establishing light paths $\mathrm{s}$ in response to a random pattern of arriving connection requests and connection holding times. This algorithm is very efficient in calculation and reduction of the blocking probability.

WLCR_FF Routing Algorithm

Step 1: Calculate every node pair (p);
For $1<\mathrm{p}$;

Step 2: Calculate K routes;

For $\mathrm{K}>1$;

Step 3: Calculate blocking probability,

For (blocking probability to be minimum) While (blocking probability is not $=1$ )

Step 4: Select a route K1;

Where $\mathrm{K} 1$ belongs to $\mathrm{K}$;

Step 5: Calculate blocking probability;

If (blocking probability $=1$ )

Route blocked

\subsection{Shortest Path Routing Algorithm}

In Shortest Path Routing for each unit of traffic, the shortest feasible path where the length of a path is defined as the number of links in it.

\section{Shortest Path Routing Algorithm}

Step 1: Choose a Source to Destination pair $p$ of the set SD pairs that have not been routed yet.

Step 2: For all paths $\mathrm{P}$ (paths that correspond to the SD pair p), find the shortest path that has not been congested.

Step 3: Route all the traffic demand of $\mathrm{p}$ through this path until either all the traffic demand for $\mathrm{w}$ is satisfied or the path is congested.

Step 4: Go to step2 until all the demand for the SD pair $\mathrm{p}$ is satisfied.

Step 5: Go to step1 until there are no more SD pairs left to be routed.

\subsection{Genetic algorithm (GA)}

A Genetic algorithm (GA) exploits the combination of alternative shortest paths for the given multicast requests in order to minimize the number of required split-capable nodes. A node with splitting capability can forward an incoming message to more than one output link. In this algorithm the initial population created randomly. Feasible solutions are generated by repeating appropriate genetic operators such as crossover and mutation. With constraint handling, GA selects only feasible solutions. When the stopping condition is satisfied, and then terminates the GA even though the solution may not be strictly optimal.

Step 1: Initialization: The GA starts with an initial population which is generated from a random seed. It selects an arbitrary combination of shortest paths from the table of shortest paths.

Step 2: Evaluation of fitness value: Individuals with good fitness value will be selected for the next generation.

Step 3: Selection: The selection method is used for selecting two parents to produce new individuals for the next generation .This selection leaves only those individuals with the highest fitness values in the population.

Step 4: Crossover: Crossover explores the diversity in the individuals of the population. the path crossover operator is used for exchanging the sub routes between two individuals The paths of requests in the individuals must have the same source and destination nodes to apply the crossover. 
Step 5: Mutation: The mutation procedure is applied after the crossover on each child independently. Every child gets an opportunity for changing each node according to the probability of mutation. The fitness value is evaluated for the individual after mutation

Step 6: Replacement: If the fitness value of the new individual is higher than that of the old individual or its parent, the new individual replaces them in order to maintain a fixed population size. Otherwise, the new individual is discarded and the next generation keeps the old individuals.

\subsection{MSPP Routing Algorithm (Mixed Shared Path Protection)}

In MSPP Routing algorithm defines three types of resources:

Primary resources that can be used by primary paths, Spare resources that can be shared by backup paths and Mixed resources that can be shared by both the primary and the backup paths. In this MSPP scheme, each connection is assigned a primary path and a backup path. Differing from pervious protection schemes, MSPP allows some primary paths and backup paths to share the common mixed resources if the corresponding constraints can be satisfied. Path-based protection includes two categories: dedicated path protection (DPP) and shared path protection (SPP). In a DPP, the backup paths cannot share the spare capacities in any conditions. But in a SPP, any two backup paths can share the spare capacities if their corresponding primary paths are link disjoint due to the single link failure constraints. The MSPP can save more wavelength links by sharing the mixed capacities

\subsection{Minimum Marginal Cost Routing Algorithm}

In this method, for each unit of traffic, all the feasible paths and are considered and choose the path those results in minimal increase in the overall cost. In other words, the path with the minimal marginal cost choosen, where the marginal cost of a path is defined as the sum of marginal costs of the links in the path.

Step 1: Choose an SD pair S of the set of Source to destination pairs that have not been routed yet.

Step 2: For all paths Pw (paths that corresponds to the SD pair $\mathrm{S}$ ), calculate the marginal cost of routing one additional unit of traffic. Only consider the paths that have not been congested.

Step 3: Choose the path with the lowest marginal cost.

Step4: Go to step2 until all the demand for the SD pair S is satisfied.

Step 5: Go to step1 until there are no more SD pairs left to be routed.

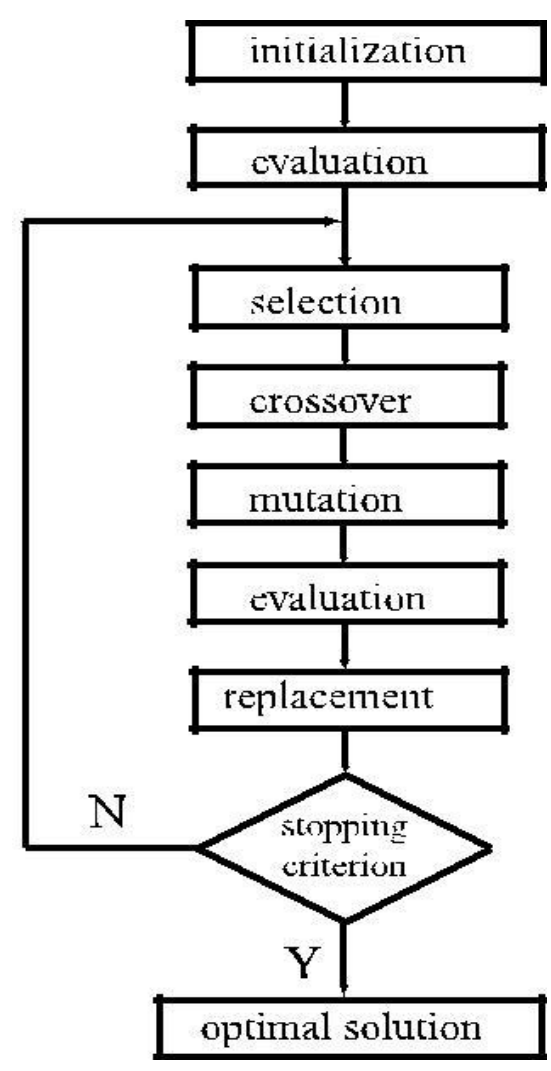

Fig 1: Flowchart of the GA procedures.

\section{PROBLEM FORMULATION}

From the literature survey it was found that the performance of the routing algorithms is calculated in terms of the blocking probability and fairness. Wavelength conversion can be used if wavelength converters are a used in the network. Wavelength conversion can eliminate the wavelength continuity constraint and can thus improve the blocking performance significantly. Thus the main task is to Proposed a Routing Algorithm that Reduce the Blocking Probability.

\section{PROPOSED WORK}

A low-complexity mathematical model has been developed which is used for the calculation of the blocking probability of network and this model does not require any simulation statistics [2]. The routing algorithm has been proposed depending upon the proposed model, which is efficient in the calculation of the blocking probability. In the proposed work a routing algorithm (improved fixed routing algorithm) is proposed. This algorithm is used for the reduction of the blocking probability and the advantage of the proposed model and algorithm is that the user has the complete knowledge of the blocking probability in advance for a particular path in the given network.

The proposed routing algorithm can be easily illustrated by the figure 2. The algorithm works in two phases:

1. In the first phase, a route for a new connection requests is selected on the basis of the shortest path. If such a route does not exist the phase 2 is performed.

2. In second phase, a route for a new connection request is selected and the path is checked for the fault if the fault exists then the next path in order of the shortest path algorithm is selected, leading to the restorable algorithm. 


\section{CONCLUSION}

In this paper the authors have simulated the behavior of various Routing Algorithms on a realistic network. The objective of this paper was to provide an overview of the architectures and the research activity of Routing Techniques in optical networks and comparison of the proposed work with the conventional algorithms. In this work a quick and efficient heuristic restorable routing algorithm is provided. Wavelength rerouting is an efficient way to improve the blocking performance. The main task of these algorithms is to achieve important gain in network blocking performance and allow an acceptable reduction in restoration.

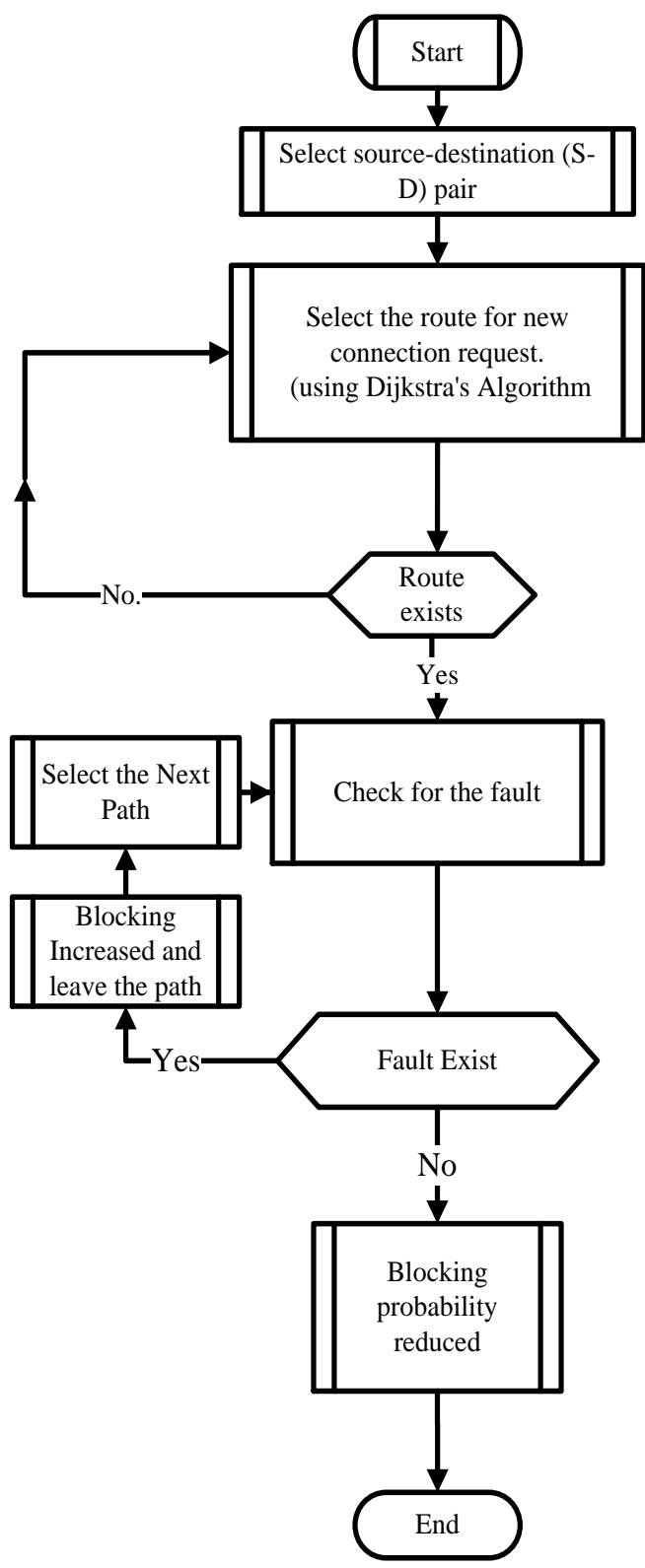

Fig 2: Proposed Algorithm

\section{REFERENCES}

[1] Sridhar Iyer and Shree Prakash Singh, "A Novel offline PLI-RWA, Regenerator Placement and Wavelength Converter Placement Algorithm for Translucent Optical WDM Networks, "Proceedings IEEE 978-1-4673-20146/2012.
[2] Amit Wason, R.S. Kaler, "Wavelength assignment algorithms for WDM optical networks, "Proceedings Optik 122 (2011) 877-880

[3] Hoa Le Minh, Zabih Ghassemlooy and Wai Pang Ng, "Investigation of Imperfect Control Pulse Effect on Performance of the All-Optical Pulse-PositionModulation Routing Scheme, "Proceedings IEEE 978-14577-0882-4/11/2011

[4] Amit Wason, R.S. Kaler, "Routing and wavelength assignment in wavelength-routed all-optical WDM networks, "Proceedings Optik 121 (2010) 1478-1486

[5] A. Drakos, T. Orphanoudakis, C. (T) Politi and A. Stavdas, "Statistical Traffic Multiplexing with Service guarantees over Optical Core Networks," Proceedings IEEE 2010

[6] Amir Askarian, Suresh Subramaniam, "Evaluation of Link Protection Schemes in Physically Impaired Optical Networks," Proceedings IEEE, 978-1-4244-34350/09/2009

[7] Yanting Luo, Yongjun Zhang, Wanyi Gu, "A New Method for Solving Routing and Wavelength Assignment Problems under Inaccurate Routing Information in Optical Networks with Conversion Capability," Proceedings IEEE 2009, SPIE-OSA-IEEE/ Vol. 76331R-1

[8] Wonhyuk Lee, kwangjong Cho, "The design of routing framework for protection \& restoration of multi-layer based on GMPLS network," Proceedings IEEE, DOI $10.1109 / \mathrm{NCM}, 2008$

[9] Xiaowen Chu, Tianming Bu Xiang-yang Li, "A Study of Light path Rerouting Schemes in Wavelength-Routed WDM Networks," Proceedings IEEE, 1-4244-0353-7/07, pp. 2400-2405, 2007'

[10] Amit Wason and Dr. R. S. Kaler , "Wavelength Assignment Problem in Optical WDM Networks," Proceedings IJCSNS, VOL.7 No.4, April 2007.

[11] Lei Guo, Jin Cao, Hongfang Yu, and Lemin Li, "PathBased Routing Provisioning With Mixed Shared

[12] Protection in WDM Mesh Networks," Journal of Light wave Technology, vol. 24, no. 3, pp. 1129-1141, March 2006.

[13] Xiaowen Chu, Bo Li, "Dynamic Routing and Wavelength Assignment in the Presence of Wavelength Conversion for All-Optical Networks," in Proceedings IEEE/ACM Transactions on Networking, VOL. 13, NO 3, JUNE 2005

[14] Xiaowen Chu and Jiangchuan Liu, "DLCR: A new adaptive routing scheme in WDM mesh networks," in Proceedings of IEEE ICC, vol. 3, pp. 1797-1801, 2005.

[15] Anwar Alyatama, "Wavelength decomposition approach for computing blocking probabilities in WDM optical networks without wavelength conversions," in Proceedings Computer Networks 49 (2005) 727-742.

[16] Johannes Hamonangan, Yongbing , Hideak ,"Optimal Multicast Routing Using Genetic Algorithm forWDM Optical Networks," in Proceedings IEICE TRANS. COMMUN., VOL.E88-B, NO.1 JANUARY 2005 
[17] P. H. Ho and H. T.Mouftah, "Shared protection inWDM mesh networks," IEEE Commun. Mag., vol. 42, no. 1, pp. 70-76, Jan. 2004.

[18] YooYounghwan, SanghyunAhn and Chong Sang Kim, "Adaptive Routing Considering the Number of Available Wavelengths in WDM Networks", IEEE Journal on Selected Areas in Communications, vol. 21, no. 8, pp. 1263-1273, October 2003.

[19] R. Ramamurthy and B. Mukherjee, "Fixed-Alternate Routing and Wavelength Conversion in Wavelength-
Routed Optical Networks," IEEE/ACM Transactions on Networking, vol. 10, no. 3, pp. 351-367, June 2002

[20] G. Mohan, C. Siva Ram Murthy, Arun K. Somani, "Efficient Algorithms for Routing Dependable Connections in WDM Optical Networks", IEEE/ACM TRANSACTIONS ON NETWORKING, VOL. 9, NO. 5, OCTOBER 2001

[21] Arakawa, S., Murata, M., and Miyahara, H.;"Design methods of multiplayer survivability in IP over WDM networks" ,In Proceeding Opti-Comm. TX. October, 2000 . 